\title{
Towards the implementation of Computer-Aided Semiosis
}

\author{
A.E. Lascu, S.C. Negulescu, C. Butaci, V. Cret
}

\author{
Alina E. Lascu, Sorin C. Negulescu \\ Lucian Blaga Univeristy of Sibiu, Romania \\ E-mail: alina.lascu@ulbsibiu.ro, sorin.negulescu@ulbsibiu.ro \\ Casian Butaci, Vasile Cret \\ Agora University, Oradea and R\&D Agora Ltd. \\ Cercetare Dezvoltare Agora Oradea, Romania \\ E-mail: casian12@yahoo.com, vcret@univagora.ro
}

Motto: In the beginning was the Word

Bible, John 1:1

\begin{abstract}
Computer-Aided Semiosis (CAS) is a concept coined by a team of researchers a couple of years ago. Since it is a promising domain due to the fact that responds to actual trans-cultural communication needed in the broad-band society - where often the message behind the words does not come clear - the subject ought being inquired more detailed as promised in other papers of the same authors. This interesting idea was inspired from Eco's theory of communication which states that the receiver "fills the message with significance"; hence it is vital for any communication and is strongly dependent on the cultures involved. In line with Eco's theory, the research in this area must be trans-disciplinary and anthropocentric. In the intention of narrowing the existing gap between the technological offers and user expectations the macro-architectural feature is that translation will progress from textual, semantically correct, to multimodal, culturally adequate, based on common concepts and "grammar" (rules to combine them into meaningful sentences); thus, this paper will present possible approaches towards the implementation of CAS. Given the fact the ontologies are considered to be the pillars of Semantic Web but also a key tool in implementing CAS, both will be a subject of this paper in the light of finding an implementation solution.

The paper is structured on five sections: the first will present the defining aspects of the concept relating it with previous research; the second section will deal with CAS approach and architecture, following with the state of the art regarding ontologies and their relation with Semantic Web. Among the conclusions, one is already noticeable: CAS could not be possible without a trans-cultural ontology.

Keywords: Computer-Aided Semiosis (CAS), Human-Computer Interaction (HCI), Ontologies, Semantic Web, Interfaces.
\end{abstract}

\section{Introduction}

The key to effective online cross-cultural communication is a well-designed transcultural ontology which to help in disambiguating between concepts that seem alike but their meaning differs from culture to culture; this tool is intended to be developed in the following years by a team of young researchers in order to substantiate and implement a new and innovative concept in the field of HCI: computer-aided semiosis (CAS). 
University of Colorado, USA developed a study regarding cross-cultural communication strategy and came up with the conclusion that often intermediaries who are familiar with both cultures can be helpful in cross-cultural communication situations. They can translate both the substance and the manner of what is said. For instance, they can tone down strong statements that would be considered appropriate in one culture but not in another, before they are given to people from a culture that does not talk together in such a strong way. They can also adjust the timing of what is said and done. Some cultures move quickly to the point; others talk about other things long enough to establish rapport or a relationship with the other person. If discussion on the primary topic begins too soon, the group that needs a "warm up" first will feel uncomfortable. A mediator or intermediary who understands this can explain the problem, and make appropriate procedural adjustments [4].

The results of this study can be as well applied in ICT due to the fact that online cross-cultural communication could also use virtual intermediaries which to have access to a transcultural ontology assisting thus the user grasping the right meaning of a certain message, i.e. in written (chat), spoken (voice) and/or visual form.

In the light of the earlier scan, this paper will present possible approaches towards the implementation of CAS. Since the ontologies are thought to be the pillars of the Semantic Web but also an important tool in implementing CAS, both will be key-subjects of this paper in the quest of finding an implementation solution.

The paper is structured on five sections: the second will present the defining aspects of the concept relating it with previous research; the third will deal with CAS rational and approach, following with the state of the art regarding ontologies and their relation with Semantic Web. Among the conclusions, one is already easily remarked: at the online level, communication can be impaired not only by the cultural differences but also by a wide range of differences such as race, age, sex, profession, religion or disabilities; in this regard, if the transcultural ontology will prove its efficiency in disambiguating cultural concepts, consequently other ontologies could be also implemented for aiding the online communication process between different users.

\section{Defining the Concepts in line with History}

According to modern paradigms, the goal of using ICTs is "obtaining a service from a huge palette of available ones" and the means is "interacting with an entity". The "entity" is either a human (e.g. when speaking via mobile phones) or a device (e.g. when buying travel documents via computers) [1]. Therefore there are three possible ways of communication as described in [1]: a) "face to face", b) "face to interface", c) "interface to interface" in the near future context of semantic web, domain ontologies and so forth.

very agent metaphor. Thus, when users employ agents (in whatever domain of activity) they expect: a) personalization (agents act considering the specific momentary needs of their clients); b) authorization (agents act on behalf of their clients, within the limits stipulated by the hiring agreement); and c) competence. In short, the agent metaphor suggests that "I hire an agent when I do not have enough time or lack competence to handle the problem myself" [1]. As regards the interface agents, the emphasis is on the interface, entailing that the agent remains hidden (i.e., the users perceive just a "smarter functionality", no "pseudoavatars" [10] intervening in a human-to-human dialogue) [1]. Considering these features, one can say that an agent, or more specific an interface agent can act like a smart mediator which to have access to knowledge (i.e. ontology) and by using it efficiently to "translate" to the user only the meaningful messages, saving a lot of time that otherwise would have been wasted. In the 21st century time is money therefore it is vital mostly in the business sector but not restricted to.

Online communications nowadays means more than a showy website and a newsletter. If 
new web-based technologies are joint with a society that is rapidly getting to think of online interactions as just as authentic as face-to-face ones, one has the possibility of radically easing the communication even in difficult (but frequent) circumstances, like those involving crosscultural interaction ( [2], [6], [7], [8], [9], [10], [11], [12], [13]) as debated and probed in previous paper of the authors.

In our previous researches we approached the transcultural interfaces' subject which could be explained in human-to-human communication as a progression from textual (semantically correct) conversions to multimodal (culturally adequate) ones [2], based on the concept of CAS. Since at this research stage the experimental models presented in the related papers are not agent-oriented, the reference to "agents" is found only in [1] and regards conceptual aspects as well as future development.

Thus far, the emphasise in our research was put on transcultural which represents the ability of people belonging to different cultures to communicate efficiently preserving in the same time their cultural identity [2]. This concept will be further on related with ontology which together, i.e. transcultural ontology, will embody the tool by which CAS could be validated as a possible new and challenging domain of HCI.

\section{Rationale and Approach}

As depicted from the title this paper intends to draw the sketch of what it should be the onward study on the implementation/ validation of CAS. Since the prime motives because of which we started this endeavour was already stated in the previous papers of this team, it is redundant to re-state them; instead this paper is set to focus on the approach and methods.

Since CAS was designed from an anthropocentric perspective, meaning to provide an assistance (i.e. an interface agent which to access a transcultural ontology, transfering the user only meaningful messages), lessening the linguistic hurdles (such as the traduttore-traditore effect), the logocratic pressure of (spoken or written) text, response time criticality, as well as the danger of distortions and noise, via a major upgrade in communication granularity: (one) idea instead of (many) words [9].

The ongoing study should be approached from a trans-disciplinary perspective, in respect with both humanists (i.e. linguists, psychologists) and technologists (i.e. ontology, interface designers and so on). When creating the ontology, the designer should bear in mind the way users and agents may "think"; how an user creates meaning from a piece of image of some sings (i.e. words) and how an interface agent does the same job effortlessly on the contrary. Though, do an agent depict the meaning same accurate as a human does? Or maybe detecting the true meaning of a message is even more endangered by human's scrambled mind which is very much contextual, in opposition with an interface agent which will act and respond based only on the given ontology and some very specific rules, and hence the probability to fail giving the expected meaning will be smaller. These are all questions which can not be answered at this point, some answers will be empirically uncovered, some may prove to be exactly contradictory with those thought in the first place. After all, this is what academically we call exploratory research. The approach will be adapted step by step base on the further findings.

\section{Ontology. State of the art}

People are able to use the Web in order to complete tasks such as finding the Icelandic word for "alphabet", reserving a plane ticket, or searching for a low price for an e-book. Still, computers cannot perform the same tasks without human direction because web pages are designed to be 
read by people, not machines. The semantic web is a vision of information that is understandable by computers, so that they can perform more of the dull work involved in finding, sharing, and combining information on the web [3].

The vision of Tim Berners-Lee regarding semantic web [3]: "I have a dream for the Web (in which computers) become capable of analyzing all the data on the Web - the content, links, and transactions between people and computers. A 'Semantic Web', which should make this possible, has yet to emerge, but when it does, the day-to-day mechanisms of trade, bureaucracy and our daily lives will be handled by machines talking to machines. The 'intelligent agents' people have touted for ages will finally materialize" is related in this study with Eco's semiosis theory (which states that the receiver "fills the message with significance" [5]), meaning that on the same features' as semantic web's CAS could be implemented but using instead a dedicated/personalized ontology based on which to develop the "translation process".

An ontology is a formal representation of a set of concepts within a domain and the relationships between those concepts [17]. The ontology envisioned by this research team is based on the idea of Maya script (a logographic type of script which used both logograms and syllabic characters [16]) by replacing, where possible, the words with images as in the catchphrase: "a picture it is worth a thousand words". Figure 1 illustrates the way the correspondence between a word-based ontology and an image-based ontology can be created, of course, the example given in this paper is very much simplified. The demarcation line was traced in order to separate the abstract and the concrete synonyms of the word apple. On one side of the line there can be easily remarked every-day interpretations of the word apple, which can without any doubt be recognized by anyone. On the other side, the other interpretations depict more abstract representations of the word apple which require a higher level of knowledge in order to be grasped, i.e. the apple polisher, the temptation (religiously), Wilhelm Tell representation (historically), Newton's gravitation theory (physical sciences), "an apple a day keeps the doctor away" (health idiom); the point is that the ontology must be trans-disciplinary and trans-culturally created in order to gather all the possible meanings and definitions for a word/concept. Disambiguation can further on continue and the figure presented above be expanded with other concepts and relation between them. An example at hand for expanding the ontology would be adding other characteristics for the fruit apple such as variety name (e.g. Granny Smith, Pink Lady, Red Delicious, Golden Delicious and so on), colour (e.g. red, green, pink, yellow), taste (e.g. sour, sweet, etc.). Also, in order to disambiguate AppleŽ's logo there can be made a separation between the old and the new logos. An important disambiguation which must be made in the first place is between English word apple and Swedish word äpple which happens to mean the same thing but they belong to different cultures which can generate later on other misunderstanding problems.

Briefly, the implementation of CAS will follow this ontology framework where the icon position has syntactic role (in line with ontology rules) and semantic role for CAS (to reduce the differences between "intentio auctoris" and "intentio lectoris") [2]. This kind of ontology based on visual rules can be further enhanced by using animations instead of images.

Considering this idea, the role of the interface agent will be to "translate". In German, the verb "to translate" means "übersetzen", the reason for this association is that in its most basic visualization, the German word means "to carry something from one side of the river to the other side of the river". In opposition the English word "to translate" does not immediately evoke the same image in the mind. In German, one can say: "mit der Fähre den Fluss übersetzen" which would literally mean: "to translate the river on a ferry boat". Anyway the translator so to name it must take in consideration both cultures from which to which the translation is being made; one must know that ad literam translation doesn't apply especially when a more subtle message (culturally dependent) must be transmitted, therefore the visualisation of the word "übersetzen" (to move from one side of the river to the other side of the river) leads to 


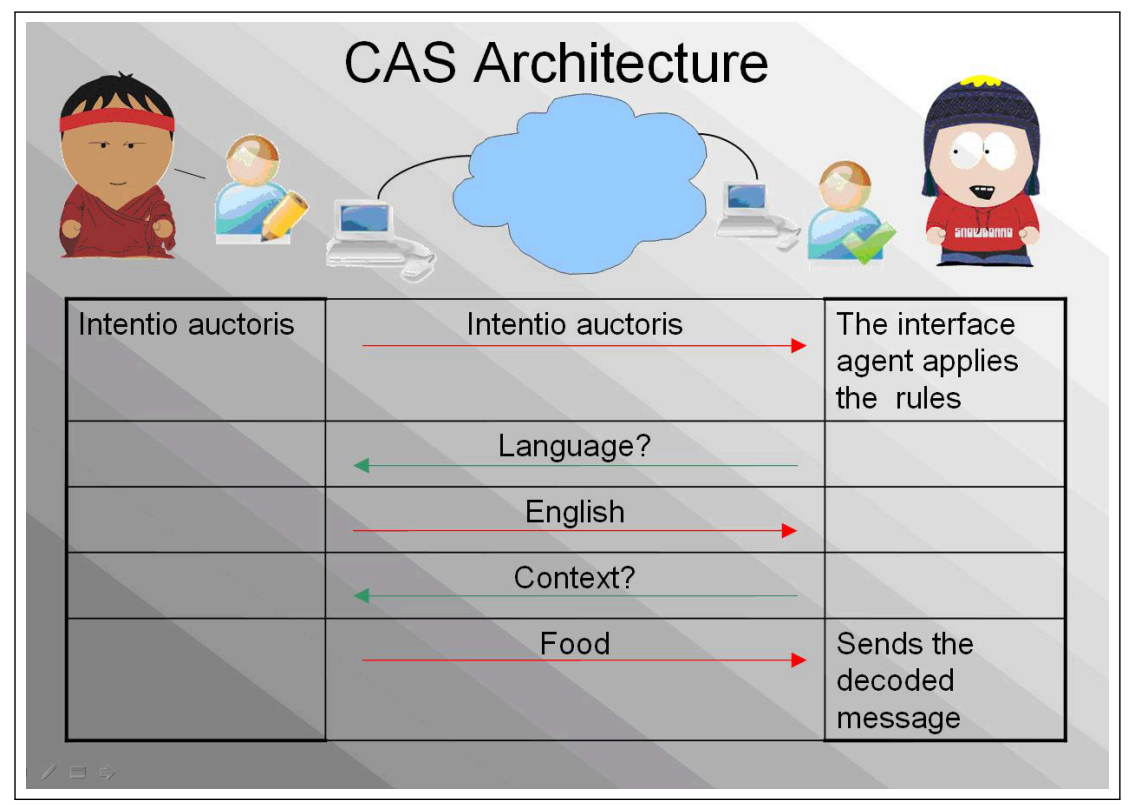

Figure 1: Example of architecture for CAS $^{1}$

several important insights into the nature of translation. In this context, the role of the agent is carrying something across the river, whether it is from here to there or from there to here. The cargo can have a multiplicity of shapes: the description of a technological object, a cultural or historical phenomenon, a poetic image, a metaphorical expression, or a human emotion, to name only a few; the parameters of each word are quite fragile; no two people will take the exact same meaning from a word [14], so the interface agent will have the hardest ever job, to extract the meaning of a message taking into consideration the transcultural ontology in line with the cultural background of both users involved in the communication process.

In order to validate CAS concept as a possible new subdomain of HCI, the researches have to focus from now on, on the implementation by creating a transcultural ontology on the framework presented in the previous section involving in this process preferable a trans-disciplinary team of researchers (linguists, psychologists, anthropologists, designers, programmers and so on) by keeping in mind the fact that an ontology deals with the nature of existence so it is a too impressive domain to be approached only by a team of thrilled researchers, willing to take the burden of exploratory research.

The future work will consider refining the ontology framework and hopefully in three-year time span (during the $\mathrm{PhD}$ studies of the first author) the objectives to be fulfilled involving European teams of researchers interested in this kind of projects.

\section{Conclusions and Future Work}

In order to validate CAS concept as a possible new subdomain of HCI, the researches have to focus from now on, on the implementation by creating a transcultural ontology on the framework presented in the previous section involving in this process preferable a trans-disciplinary team of researchers (linguists, psychologists, anthropologists, designers, programmers and so on) by keeping in mind the fact that an ontology deals with the nature of existence so it is a too impressive domain to be approached only by a team of thrilled researchers, willing to take the burden of exploratory research.

The future work will consider refining the ontology framework and hopefully in three-year 
time span (during the $\mathrm{PhD}$ studies of the first author) the objectives to be fulfilled involving European teams of researchers interested in this kind of projects.

\section{Acknowledgements:}

This research is fully supported by the POSDRU/88/1.5/S/60370 project which is co-financed by the European Social Fund through the Sectoral Operational Programme for Human Resources Development 2007-2013.

We thank Mr. Cătălin Boaru from Apple IMC Romania for kindly granting us the permission to use the Appleß logo.

Copyright notice: Apple and the Apple logo are trademarks of Apple Computer, Inc., registered in the U.S. and other countries.

Disclaimer: This article is an independent publication and has not been authorized, sponsored, or otherwise approved by Apple Computer, Inc.

Images were taken from public domain graphic sites with their own disclaimers, and a very few were taken from sites that contained no copyright information or terms of use.

\section{Bibliography}

[1] Bărbat, B.E. (2009). Interface Agents for Transcultural Communication: A Framework . In Sapio, B. (Ed.), The Good, the Bad and the Challanging. The user and the future of information and communication technologies (pp.666-675). Copenhagen: Cost Action 298.

[2] Bărbat, B.E., Negulescu, S.C., Lascu, A.E., Popa, E.M. (2007). Computer-Aided Semiosis. Threads, Trends, Threats. In Mastorakis, N.E. (Ed.), Proc. of the 11th WSEAS International Conference on Computers (pp.269-274). Agios Nikolaos, Crete: ICCOMP '07.

[3] Berners-Lee, T. (1999). Weaving the Web: The Original Design and Ultimate Destiny of the World Wide Web by its Inventor. New York: HarperOne.

[4] Conflict Research Consortium (1998). Cross-Cultural Communication Strategies. Retrived 2010, from University of Colorado, USA. Web site: http://www.colorado.edu/conflict/peace/treatment/xcolcomm.htm.

[5] Eco, U. (2005). The Limits of Interpretation. Bloomington, SUA: Indiana University Press.

[6] Georgescu, A.V., Lascu, A.E., Bărbat, B.E. (2008). Protensity in Agent-Oriented Systems. Role, Paths, and Examples. Int. J. of Computers, Communications \& Control, III, 304-309.

[7] Lascu, A.E., Fabian, R. (2007). e-Semiotics for Romanian-German trans-cultural interfaces. In Sapio, B. et al (Ed.), The Good, the Bad and the Unexpected: The User and the Future of Information and Communication Technologies (pp.on CD). Moscow, Russian Federation: COST Action 298 Participation in the Broadband Society.

[8] Lascu, A.E., Georgescu, A.V. (2009). From Extensity to Protensity in CAS: Adding Sounds to Icons. In Esposito, A. et al (Ed.), Multimodal Signals: Cognitive and Algorithmic Issues (pp.130-137). Vietri sul Mare, Italy: Springer.

[9] Lascu, A.E., Moisil, I., Negulescu, S.C. (2009). Computer-Aided Semiosis mirrored in Creolization. Rational and Approach . International Journal of Advanced Statistics and IT\&C for Economics and Life Sciences, 1(1), 38-43. 
[10] Lascu, A.E., Negulescu, S.C., Cioca, M., Zerbes, M.V. (2009). Interface Agents as Virtual Tutors. Conference Proceedings of Balkan Region Conference on Engineering and Business Education \& International Conference on Engineering and Business Education, 2, 626-629.

[11] Lascu, A.E., Negulescu, S.C., Kifor, C.V. (2009). Different Time Perception in Creolization Mirrored in Transcultural Interface for "Immediate". In Sapio, B. et al (Ed.), The Good, the Bad and the Challenging - The user and the future of information and communication technologies (pp.661-666). Copenhagen, Denmark: COST Action 298 Participation in the Broadband Society.

[12] Negulescu, S.C., Lascu, A.E., Oprean, C. (2009). Cultural Differences in Decision-Making. A Transcultural Interface for Gambler's Fallacy. In Sapio, B. et al (Ed.), future of information and communication technologies (pp.656-661). Copenhagen, Denmark: COST Action 298 Participation in the Broadband Society.

[13] Prundurel, A., Negulescu, S.C., Lascu, A.E. (2007). Mini-Ontology for Trans-Cultural Interfaces. In Sapio, B. et al (Ed.), The Good, the Bad and the Unexpected: The User and the Future of Information and Communication Technologies (pp.on CD). Moscow, Russian Federation: COST Action 298 Participation in the Broadband Society.

[14] Rainer Schulte (1999). The translator as mediator between cultures. Retrived 11.2009, from Translation Studies. Web site: http://translation.utdallas.edu/translationstudies/mediator_essay1.html.

[15] Russell S., Norvig P. (2003). Artificial Intelligence: A Modern Approach. NJ: Prentice Hall.

[16] Simon Ager (2009). Mayan script. Retrived 2010, from Omniglot - writing systems and languages of the world. Web site: http://www.omniglot.com/ writing/mayan.htm.

[17] Wikipedia (2009). Ontology (information science). Retrived 11.2009, from Wikipedia. Web site: http://en.wikipedia.org/wiki/Ontology_(information_ science). 